Check for updates

Cite this: J. Mater. Chem. C, 2017, 5, 11185

Received 8th June 2017,

Accepted 20th July 2017

DOI: $10.1039 / c 7 t c 02549 a$

rsc.li/materials-c

\title{
2D material liquid crystals for optoelectronics and photonics
}

\author{
B. T. Hogan, (D) ${ }^{\text {ab }}$ E. Kovalska, (D) ${ }^{c}$ M. F. Craciun ${ }^{b}$ and A. Baldycheva (D)*b
}

\begin{abstract}
The merging of the materials science paradigms of liquid crystals and 2D materials promises superb new opportunities for the advancement of the fields of optoelectronics and photonics. In this review, we summarise the development of 2D material liquid crystals by two different methods: dispersion of 2D materials in a liquid crystalline host and the liquid crystal phase arising from dispersions of 2D material flakes in organic solvents. The properties of liquid crystal phases that make them attractive for optoelectronics and photonics applications are discussed. The processing of $2 \mathrm{D}$ materials to allow for the development of 2D material liquid crystals is also considered. An emphasis is placed on the applications of such materials; from the development of films, fibers and membranes to display applications, optoelectronic devices and quality control of synthetic processes.
\end{abstract}

\section{Introduction}

Two-dimensional (2D) nanocomposite materials with dynamically tunable liquid crystalline properties have recently emerged as a highly-promising class of novel functional materials, opening new routes within a wide variety of potential applications from

${ }^{a}$ EPSRC Centre for Doctoral Training in Electromagnetic Metamaterials, University of Exeter, EX4 4QL, UK

${ }^{b}$ Department of Engineering and Centre for Graphene Science, College of Engineering, Mathematics and Physical Sciences, University of Exeter, Exeter, EX4 4QF, UK. E-mail: a.baldycheva@exeter.ac.uk

${ }^{c}$ Laboratory of Smart Materials and Devices, Department of Physics, Bilkent University, Ankara, 06800, Turkey the deposition of highly uniform layers and heterostructures, to novel display technologies. Here, we will introduce the underlying concepts that underpin this recent technological advance; provide an overview of the synthetic routes towards such $2 \mathrm{D}$ nanocomposite materials; and review recent advances in the application and applicability of these materials within the fields of optoelectronics and photonics.

Since the advent of graphene in $2004,{ }^{1}$ there has been an explosion in the investigation of a wide range of atomically thin (two-dimensional) materials. In addition to graphene (exfoliated from graphite), materials that can be reduced to monolayer size have been shown to include: graphene oxide (from graphite oxide); transition metal dichalcogenides (TMDCs) - for example

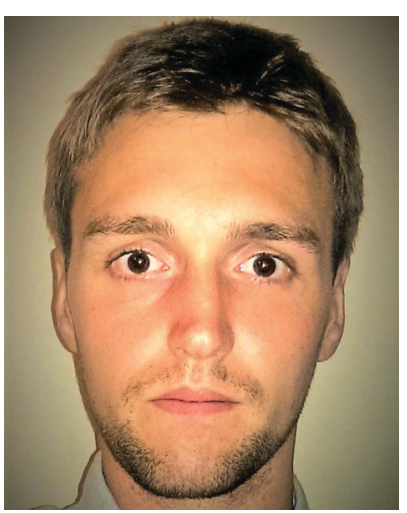

B. T. Hogan
Ben graduated from the University of Bath in 2015 with a MSci in Natural Sciences. He is currently a postgraduate researcher within the EPSRC Centre for Doctoral Training in Metamaterials at the University of Exeter, UK, working in the Optoelectronic Systems Group lead by Prof. Baldycheva. His research focuses on the synthesis, characterisation and application to devices of novel optoelectronic and photonic $2 D$ liquid crystal materials incorporating metamaterial structures.

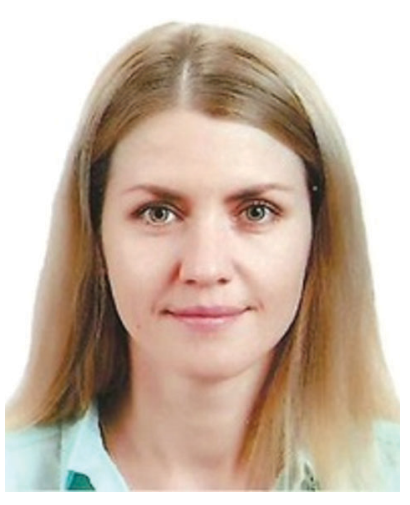

\section{E. Kovalska}

Dr Evgeniya Kovalska is a Postdoctoral Research Fellow in the Laboratory of Smart Materials and Devices at Bilkent University of Ankara, Turkey. Her current research pertains to synthesis and application of graphene, transfer printing processes, patterning methods, developing new micro/ nano-scale devices, chemical and electrooptical characterization, and microfabrication techniques. She obtained her PhD degree in chemistry with specialty in the

physics and chemistry of surfaces in the Department of Physical Chemistry of Carbon Materials at Chuiko Institute of Surface Chemistry of the NAS of Ukraine, Kyiv. 
$\mathrm{MoS}_{2}, \mathrm{WSe}_{2}$ and $\mathrm{MoTe}_{2}$; and hexagonal boron nitride (h-BN) amongst countless others. The possibilities for applications of these materials are almost limitless, owing to the diverse properties that they exhibit. However, adoption of these materials in novel optoelectronics and photonics applications is often limited by challenges surrounding the scalability, cost of production processes or limited device tunability. Recently, two paradigms of significant interest for the development of novel functional materials where dynamic reconfigurability is delivered through the exploitation of liquid crystalline properties and 2D materials have emerged. Firstly, 2D material particles can be dispersed in a conventional liquid crystal host. ${ }^{2,3}$ Alternatively, 2D materials dispersed in specific solvents have been shown to display lyotropic liquid crystalline phases within certain ranges of 2D material concentration. $^{4,5}$

\section{Liquid crystals}

The liquid crystal phase is a phase of matter that exists for a variety of molecules and materials, depending on their geometric and chemical properties, with characteristics intermediate to those of a conventional crystalline solid and a liquid., ${ }^{6,7}$ Liquid crystals (LCs) have found use in a variety of applications through the years (Fig. 1). The liquid crystal phase was initially described by Austrian botanist Friedrich Reinitzer in 1888 when looking at the properties of cholesterol derivatives, ${ }^{8,9}$ although some credit also goes to Julius Planer, who reported similar observations 27 years prior. ${ }^{10,11}$ This new and distinct state of matter was then identified as the "liquid crystal phase" by Otto Lehmann in 1890 and in 1904 the first commercially available LCs were produced by Merck-AG. ${ }^{12}$ Over the following 18 years, scientists established the existence of three distinct liquid crystalline phases (nematic, smectic and cholesteric) ${ }^{12}$ but, with no applications of note forthcoming, the study of LCs was halted. For the next 30 years, the scientific community ignored
LC materials, considering them as an interesting curiosity. However, following a renaissance in liquid crystal science in the 1950s, the previously scientific curiosity has become a ubiquitous part of the modern technology landscape.

During the 1950s, the invention of the first cholesteric LC temperature indicators, as well as advances in analytical metrology, cancer diagnostics and non-destructive material testing methods drove a new era in liquid crystal science. By 1962, liquid crystals were already finding applications in state-of-the-art laser devices, despite the relative youth of laser science. However, the most important technological innovation came in 1965 with the development of the first LC displays (LCDs). ${ }^{13-15}$ Subsequently, twisted nematic LCDs (1969-1971) advanced the field further. ${ }^{13-15}$ Significant breakthroughs in the evolution of liquid crystal technologies occurred in the 1980-1990s and continue to have a profound impact on day-to-day life: the miniaturisation of display technologies facilitated the development of portable PCs, mobile telephones and countless other innovations. ${ }^{13-15}$ Since the start of the new millennium, LCs and recently discovered $2 \mathrm{D}$ material LCs have come into demand as optoelectronic and photonic materials. ${ }^{3,16-19}$

The possibility of the existence of a liquid crystal phase stems principally from the geometric structure of the molecules in the material as well as the functional groups present in the molecule. In lyotropic liquid crystals, mesogens are dispersed in a host solvent (typically water but other organic solvents can be used depending on the molecule). ${ }^{6,7,20}$ Lyotropic liquid crystals exhibit a liquid crystal phase within a certain range of temperatures but also require a concentration of the active mesogens that falls within a certain range. In the lyotropic phase, the fluidity of the material is induced by the solvent molecules rather than being intrinsic to the mesogens themselves. The mesogens contain immiscible solvophilic and solvophobic parts separated at opposing 'ends' or facets of the molecule, making them amphiphilic.

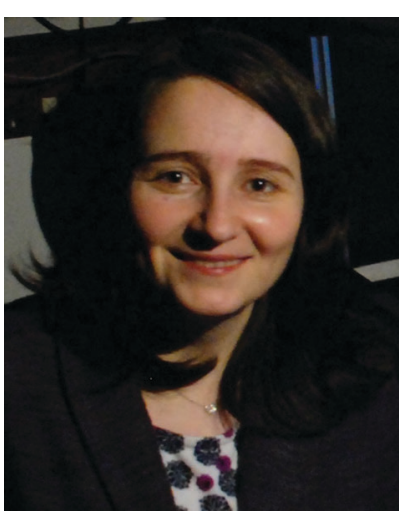

M. F. Craciun
Monica Craciun is a Professor in Nanoscience and Nanotechnology. She received her $P h D$ from Kavli Institute of Nanoscience, Delft University of Technology, The Netherlands and held a prestigious Fellowship of the Japanese Society for the Promotion of Science at The University of Tokyo, Japan. Her research is focused on twodimensional materials for emerging technologies. She has published more than 80 papers in reputed journals such as Nature Nanotechnology, Science Advances, Advanced Materials, and Nano Letters. She was awarded an early career fellowship from the U.K. Engineering and Physical Sciences Research Council and was investigator on more than 30 funded research projects.

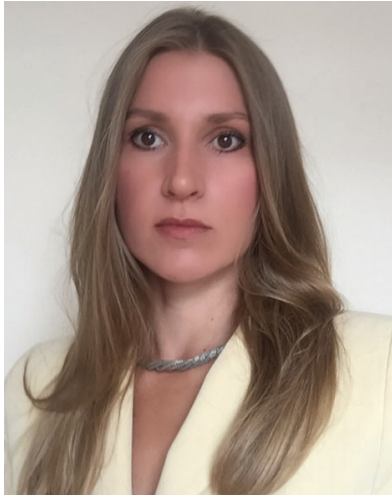

A. Baldycheva
Prof. Anna Baldycheva completed her BSc(Hons) at Saint-Petersburg University in 2008 and $P h D$ at Trinity College Dublin in 2012. She is currently leading a highly interdisciplinary research group, Opto-Electronic Systems Laboratory, at the Centre for Graphene Science at the University of Exeter. Prof. Baldycheva's research group works in the areas of Si photonics, and flexible and fluid optoelectronics. Her research interests span from the development of the new photonic composite materials to the engineering of integrated hybrid electronic-photonic devices for application in communications, energy harvesting, and bio-chemical sensing. Since 2011 she has over 50 peer-reviewed publications, invited talks and conference proceedings. 


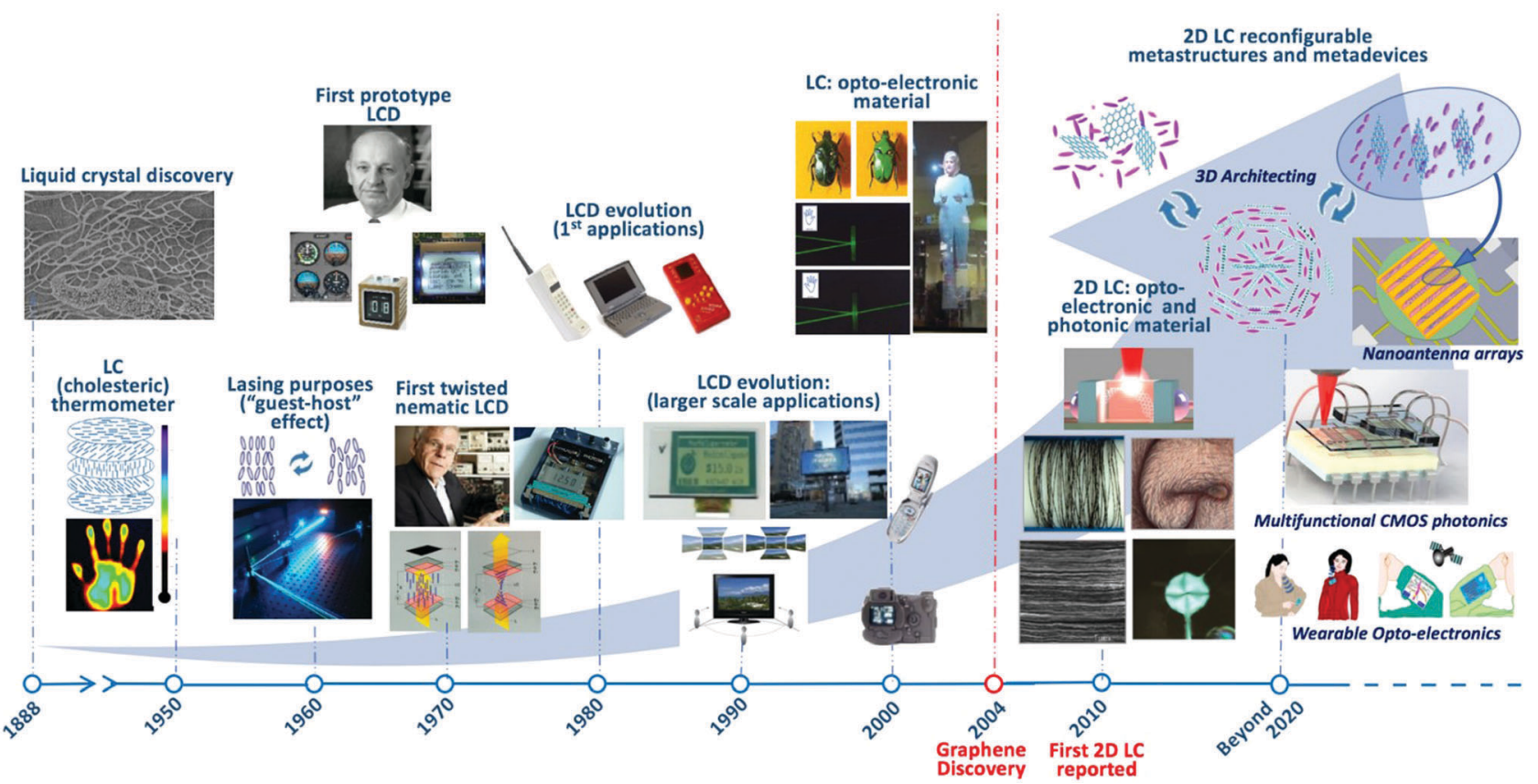

Fig. 1 Timeline of the history of liquid crystal phase applications, from their discovery to the present day. 1888: the LC phase was first reported by Friedrich Reinitzer (image reproduced with permission from Mitov, ChemPhysChem, 2014, ${ }^{96}$ (C)2014 Wiley-VCH)). 1950-1959: the development of the first cholesteric phase and LC thermometers. 1962: the switchable orientation of LC molecules was first utilised in laser devices. 1965: the first prototype LCD was developed by George H. Heilmeier before finding limited applications. 1969-1971: the first twisted nematic cell displays were developed from the initial work of Martin Schadt and incorporated in devices. 1980-1990s: LCDs found widespread application in small mobile devices as the display of choice. From the 1990s onwards, much larger LCDs have appeared while advances in the technology have allowed the production of high resolution small-scale displays. Beginning in the new millennium, LCs have emerged as desirable optoelectronic materials. They have, as such, been studied from natural sources such as can be found in beetles, as well as in polarisation-selective waveguiding and in holography. The discovery of graphene in 2004 opened up new avenues for LC science and, since 2010, the emergence of LCs combined with 2D materials has opened applications in developing 2D material fibers (reproduced with permission from Xu and Gao, Nat. Commun., $2011^{4}$ (C)2011 Nature Publishing Group)), reflective displays (reproduced from He et al., Nanoscale, $2014^{52}$ with permission from The Royal Society of Chemistry (C2014), deposition of uniform layered structures (reproduced with permission from Jalili et al., ACS Nano, $2013^{78}$ (C)2013 American Chemical Society)) and as a platform for novel optofluidic devices (reproduced with permission from Hogan et al., Sci. Rep., $2017^{3}$ (C) 2017 Nature Publishing Group)). The future of the field promises to revolutionise fields from CMOS photonics, to metastructures and metadevices and wearable technologies. All images utilised under a free-to-use creative commons license except where otherwise credited.

As one end has a preferential interaction with the host solvent, ordering of the amphiphilic molecules occurs to maximise the solvophilic 'head' interaction with the host solvent while minimising that for the solvophobic 'tail'. The structures formed by the mesogens are dependent on the relative volumes of the 'head' and 'tail' as well as the concentration of the molecules within the solvent.

At very low concentrations, there will be no ordering of the amphiphilic molecules dispersed in the solvent. ${ }^{6,7,20}$ As the concentration is increased, there will be a critical concentration at which micelles are spontaneously formed - however, the micelles do not order themselves so this still does not represent a liquid crystal phase. At higher concentrations, the micelles must order themselves as the inter-micelle interactions become energetically important above a critical micellular concentration within the solvent. Typically, a hexagonal columnar phase is formed where long cylindrical rods of amphiphilic mesogens arrange themselves into a hexagonal lattice structure but other structures are possible depending on the mesogen. As the concentration increases further, a lamellar phase will form, with layers of the mesogens separated by thin layers of solvent. In lyotropic liquid crystals, it is objects formed by the aggregations of amphiphiles that can then be ordered in the same ways as observed for thermotropic liquid crystals. Lyotropic liquid crystals possess significant tunability as the structural properties are highly sensitive to changes in concentration. For example, within in the hexagonal columnar phase, the lattice parameters can be varied by varying the solvent volume in the mixture.

Liquid crystals are of particular interest due to their inherent ordering while in the liquid phase and for their ability to align the director along an external field. ${ }^{21,22}$ Permanent electric dipoles can exist in the individual liquid crystal molecules when one part of the mesogen has a positive charge while another has a negative charge. When an external electric field is then applied to the liquid crystal, the dipoles orient along the direction of the field as the electric field exerts a force on the dipoles. Some liquid crystal molecules, however, do not form a permanent dipole but can still be influenced by an electric field. The shape anisotropy of many liquid crystal mesogens means that they are highly polarisable and as such an applied electric field can induce a dipole by relocating the electron density within the molecule. While not as strong as permanent dipoles, orientation of the induced dipoles 
with the external field still occurs. The effects of magnetic fields on liquid crystal molecules are analogous to electric fields with the molecules aligning with or against the magnetic field.

\section{D materials}

Whereas, in the past, 2D materials have typically been produced by either a mechanical cleavage $\operatorname{method}^{1}$ or by vapour deposition, ${ }^{23-26}$ recently liquid phase exfoliation has attracted significant interest due to the inherent scalability of the process. Liquid phase exfoliation (Fig. 2) is a method where a bulk material is dispersed in a solvent and then layers are broken apart. ${ }^{27-31}$ In most cases, the layers are broken apart using ultrasonication where high frequency sound waves are transmitted through the solution. ${ }^{27-30,32-35}$ The sound waves induce the formation of bubbles and cavities between layers which break the layers apart as they expand. However, they also cause strains in the material which cause intralayer cleavage of the particles, reducing the size of the particles obtained after exfoliation. Other than ultrasonication, other methods have been developed for liquid phase exfoliation, including strong acid induced oxidation reactions causing cleavage $e^{36}$ and freezing of water intercalated layered structures where expansion of water as it freezes causes interlayer cleavage. ${ }^{37}$ Following exfoliation, particles of specific sizes can be isolated by centrifugation of the dispersion, ${ }^{38,39}$ solvent induced selective sedimentation ${ }^{40}$ or by $\mathrm{pH}$-assisted selective sedimentation ${ }^{41}$ amongst others. Materials of interest for optoelectronics and photonics that can be reduced to few-layer or monolayer by means of liquid phase exfoliation encompass a broad range; from graphene and its derivatives to transition metal dichalcogenides (TMDCs), metal oxides and hexagonal boron nitride (h-BN) amongst many others. Liquid phase exfoliated 2D materials are of significant interest for the production of $2 \mathrm{D}$ material liquid crystal composites as the exfoliating solvent can be used as the fluid host for spontaneous liquid crystal phase self-assembly, ${ }^{4,27,42-53}$ or to allow combination with conventional liquid crystals. ${ }^{3}$

Amongst materials discussed further here, graphene can be exfoliated from bulk graphite owing to the weak van der Waals interactions between layers in graphite. ${ }^{54}$ Graphene is an allotrope of carbon consisting of a two-dimensional hexagonal lattice with a single carbon atom at each vertex. The carbon atoms in graphene are $\mathrm{sp}^{2}$ hybridised in-plane with these $\mathrm{sp}^{2}$ electrons forming three carbon-carbon bonds. The final $\mathrm{p}$ orbital is unhybridised and directed out of the plane. For a graphene

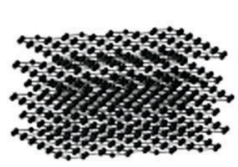

Starting material

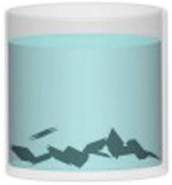

Dispersion in solvents

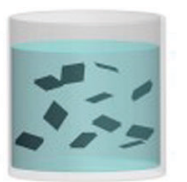

Ultrasonication

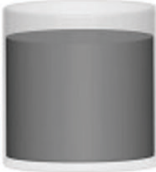

Dispersion after Ultracentrifugation
Fig. 2 Liquid phase exfoliation of 2D materials. (a) Starting material (e.g., graphite), (b) chemical wet dispersion, (c) ultrasonication and (d) final dispersion after the ultracentrifugation process. Reproduced with permission from Bonaccorso and Sun, Opt. Mater. Exp., 2014 ${ }^{31}$ (C2014, The Optical Society). sheet these out of plane $\mathrm{p}$ orbitals hybridise to form the delocalised $\pi$ and $\pi^{*}$ bands which are responsible for graphene's exceptional electronic properties; these exceptional properties make graphene of significant interest as a material for forming electrical contacts, films and fibers.

Graphene oxide is the 2D material produced by the exfoliation of graphite oxide. ${ }^{55}$ Maximum oxidation of graphite results in a carbon to oxygen ratio between 2.1 and 2.9. Graphite oxide retains the layered structure of graphite but the interlayer spacing is increased and no longer regular for bulk graphite oxide. The oxidation of graphite introduces three types of oxygen containing functional groups to the structure: epoxy bridges (oxygen bridging between two carbons on the surface of a graphitic sheet), hydroxyl groups (on either the surface or the edges) and carboxyl groups (on the edges of the graphitic sheets). ${ }^{56}$ Graphene oxide can be exfoliated from bulk graphite oxide analogously to graphene from graphite. ${ }^{54,55}$ However, the intercalation of the graphitic carbon sheets by oxygenated functional groups results in graphene oxide being more readily exfoliatable. This means that graphene oxide can be exfoliated to few layers and even monolayer in large quantities without the use of additional surfactant molecules. ${ }^{33,35}$ Graphene oxide possesses nonlinear optical properties of significant interest for applications in ultrafast photonics and optoelectronics. The saturable absorption can be used for pulse compression, mode locking and Q-switching of laser systems. ${ }^{57}$ The large observed Kerr effect introduces possibilities in all-optical switching and signal regeneration and hence optical communications devices. ${ }^{58}$ The nonlinear optical properties of graphene oxide can be tuned by controlling the carbon to oxygen ratio of the material, ${ }^{59}$ this tuning has been achieved using laser irradiance to reduce the material.

Transition metal dichalcogenides (TMDCs) are a class of material where transition metal atoms are connected by bridging group 14 elements with a stoichiometry of $1: 2$ to form layers. The layers are held together by weak van der Waals interactions and therefore present an ideal candidate for reduction to few-layer or monolayer materials. Cleavage to monolayer is typically achieved using mechanical exfoliation methods but few-layer material can be readily attained using liquid-phase exfoliation methods. Many different TMDCs have been synthesised. A common example, molybdenum disulfide $\left(\mathrm{MoS}_{2}\right)$, consists of layers of molybdenum atoms bound to six sulfide ligands in a trigonal prismatic coordination sphere. ${ }^{60,61} \mathrm{MoS}_{2}$ is an indirect bandgap semiconductor with a band gap of $1.23 \mathrm{eV}$ in its bulk form ${ }^{62}$ but the monolayer form has a direct bandgap of $1.8 \mathrm{eV}^{63}$ so can be used in switchable transistors and photodetection devices. ${ }^{60}$ $\mathrm{MoS}_{2}$ can emit light opening applications in in situ light generation devices. ${ }^{60}$

\section{D material liquid crystals}

It has been shown that by dispersing nanoparticles or molecules in a liquid crystal host that the ordering of the liquid crystal mesogens can impart ordering to the dispersed particles. ${ }^{64-66}$ The nanoparticles have been shown theoretically ${ }^{67}$ and 
experimentally ${ }^{3,68-72}$ to align with the disclinations of the liquid crystal due to the energetic favourability of such an alignment. More recently, the impartment of ordering from a liquid crystal host has also been shown with dispersed 2D material particles. ${ }^{3,73}$ Additionally, dispersions of graphene oxide in water have been shown to have a lyotropic liquid crystal phase within a specific range of concentrations of dispersed graphene oxide particles (Fig. 3), where the dispersed discotic graphene oxide particles are either stacked in the columnar manner typical of discotic liquid crystals or exhibit ordering analogous to a nematic phase. ${ }^{45,47,48}$ The liquid crystal phase of the graphene oxide dispersions arises due to the competition between the long-range electrostatic repulsion between particles, originating from ionised functional groups at the edges of the particles, and the weak attractive interactions originating from the unoxidised graphitic domains on the surface. ${ }^{74,75}$ The liquid crystallinity is therefore dependent on the particle size; more precisely to the ratio of the surface area to the circumference (and number of layers) as this determines the balance of the attractive and repulsive forces. ${ }^{75}$ Most dispersions of liquid phase exfoliated graphene oxide will consist of particles of differing sizes and therefore the polydispersity of the particles becomes an important factor. ${ }^{51}$ Additionally, this balance is affected by the degree of oxidation - the carbon to oxygen ratio of the material. ${ }^{75}$ The stability of the liquid crystal phase can also be strongly affected by the ionic content of the solvent as this determines the degree of ionisation of the oxygen containing functional groups on graphene oxide. ${ }^{43,75}$ The $\mathrm{pH}$ of the solvent also affects the critical concentration for the onset of liquid crystalline behaviour. ${ }^{76}$ By tuning these separate parameters, it is possible to observe either a nematic phase or a columnar phase of the graphene oxide dispersion (Fig. 4). The different liquid crystalline phases can be observed using photoluminescence measurements as there is a strong polarisation dependence of the photoluminescence for ordered mesophases in graphene oxide dispersions. ${ }^{77}$

Similarly, this liquid crystal phase has been observed in a range of other organic solvents including acetone, dimethylformamide, ethanol, cyclohexylpyrrolidone and tetrahydrofuran ${ }^{33,78}$ (Fig. 5). The concentration of particles required to give rise to the liquid crystal phase is different for each solvent, but there is

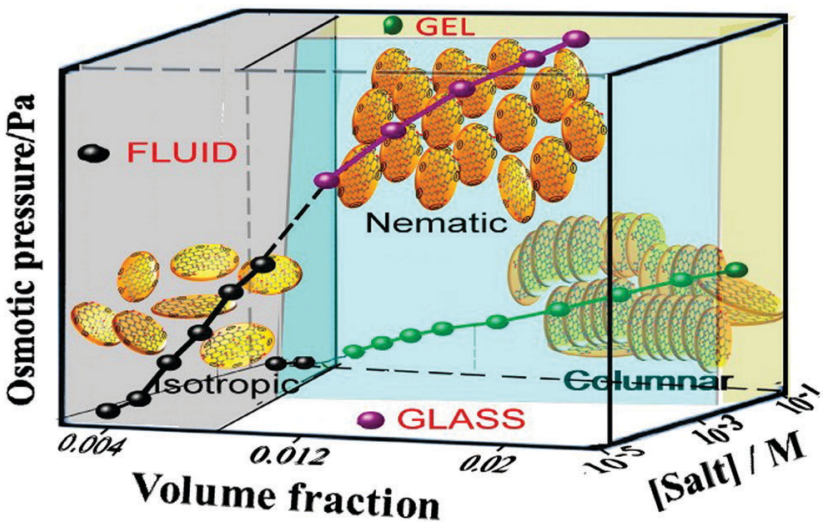

Fig. 4 Phase diagram of graphene oxide aqueous dispersions in terms of osmotic pressure, volume fraction of $\mathrm{GO}$ and salt concentration in the solution. Reproduced with permission from Konkena and Vasudevan, J. Phys. Chem. C, $2014^{75}$ (C)2014, American Chemical Society).

also some discrepancy between the threshold concentrations observed for the same solvent due to the effect of the size, shape and polydispersity of the graphene oxide particles in the solution. A liquid crystal phase has also been observed for graphene exfoliated and dispersed in chlorosulfuric acid. ${ }^{27}$ A similar phase has been observed in other solvents for graphene and small graphitic particles although only with the addition of either stabilising surfactant ${ }^{4,5,79}$ or polymer coatings. ${ }^{80}$ Dispersions of graphene in water have been reported to show an extrinsic chirality associated with a cholesteric liquid crystal phase. ${ }^{4}$ More recently a liquid crystal phase has been observed for dispersions of molybdenum disulfide at high concentration in water ${ }^{50}$ suggesting the possibility of liquid crystalline phases existing for a far greater range of dispersions of $2 \mathrm{D}$ materials. ${ }^{79}$

\section{Applications and prospects}

\section{Films, fibers, membranes and inks}

The self-assembling nature of liquid crystalline materials has led to the use of graphene oxide dispersions for the formation of well-ordered layers and stacks of 2D materials. Behabtu et al., ${ }^{27}$
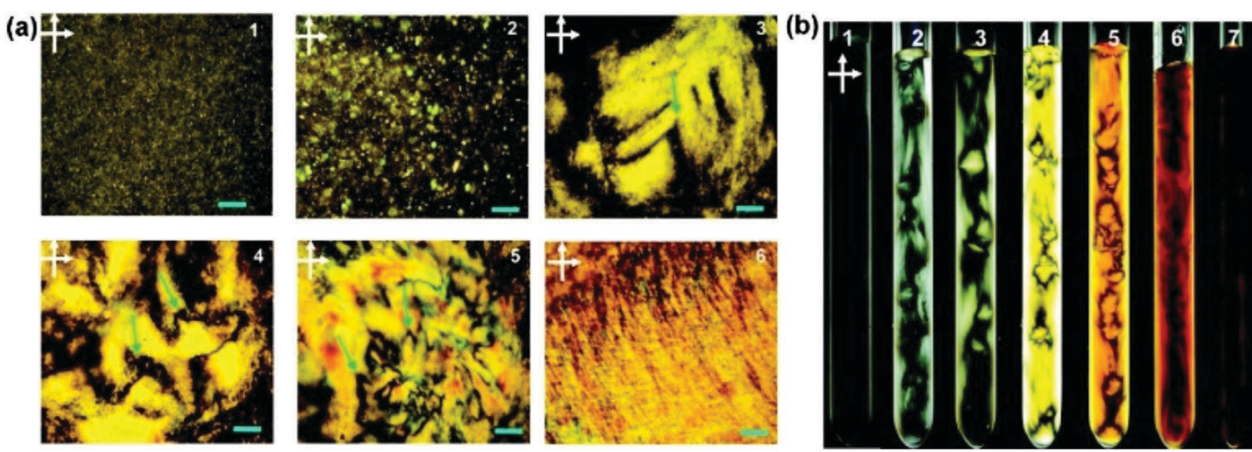

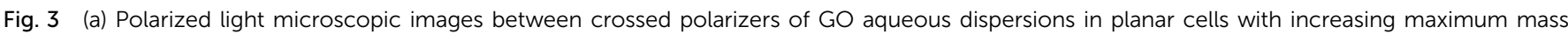

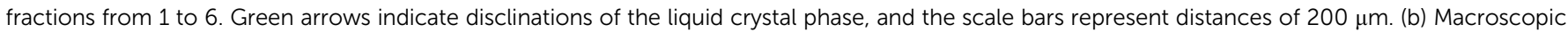

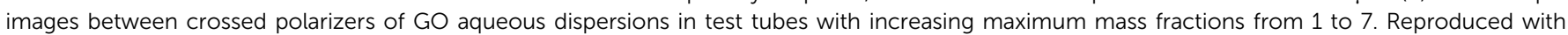
permission from Xu and Gao, ACS Nano, $2011^{47}$ (C)2011, American Chemical Society). 

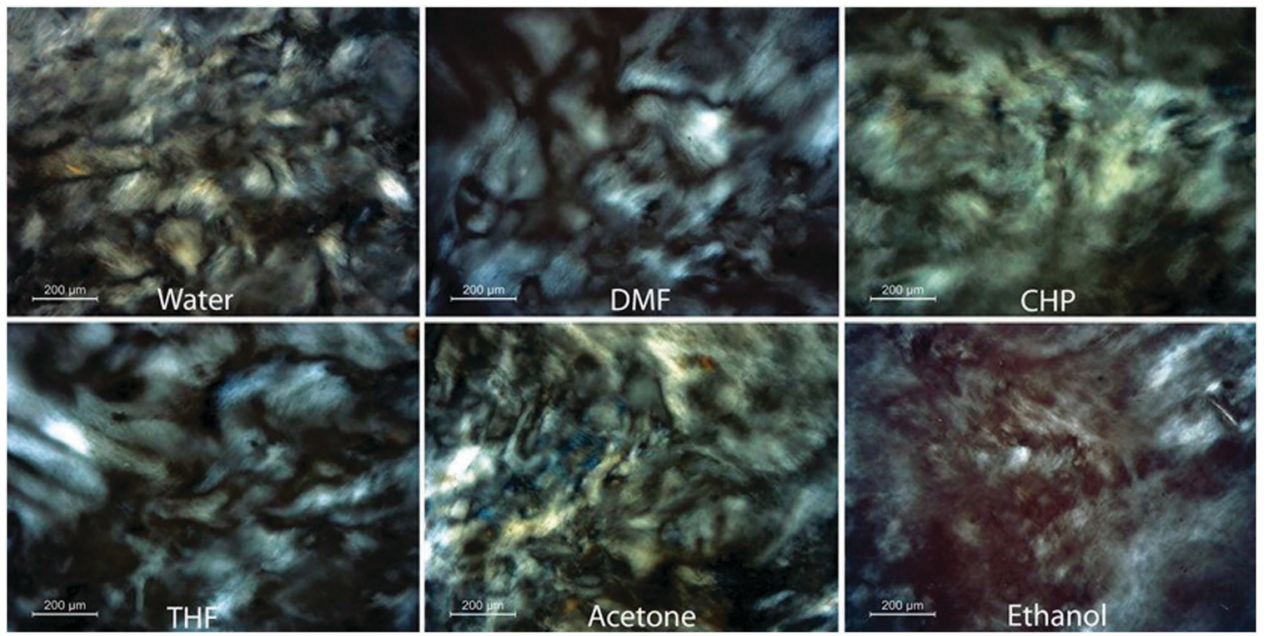

Fig. 5 Schlieren textures observed in dispersions of graphene oxide in a range of organic solvents under microscopy using crossed polarisers. Reproduced with permission from Jalili et al., ACS Nano, $2013^{78}$ (C)2013 American Chemical Society).

demonstrated that graphite spontaneously exfoliates into singlelayer graphene in chlorosulfonic acid, and spontaneously forms liquid-crystalline phases at high concentrations. Transparent, conducting films were produced from the liquid crystalline dispersions. Jalili et $_{\text {al. }}{ }^{78}$ showed that self-assembly of graphene oxide sheets is possible in a wide range of organic solvents. The prepared dispersions were employed to achieve self-assembled layer-by-layer multifunctional 3D hybrid architectures comprising SWNTs and GO with promising mechanical properties (Fig. 6). More recently, the same group has showed that similar self-assembly can be achieved using liquid crystalline dispersions of molybdenum disulfide.$^{50}$ Layers of these materials have been combined with other materials for a variety of diverse applications such as photovoltaics ${ }^{81}$ and improving the mechanical properties of composite materials,$^{82}$ the more homogeneous layers produced from the liquid crystalline dispersions are of significant interest to applications of these natures. The use of liquid crystalline dispersions of graphene oxide to produce uniform layers has been used as a precursor to forming similarly uniform structures of graphene through the reduction of the graphene oxide. ${ }^{4,49}$ Akbari et al. demonstrate that the discotic nematic phase of GO can be shear aligned to form highly ordered, continuous films of multi-layered GO on a supporting membrane. The highly ordered graphene sheets in the plane of the membrane make organized channels and give greater permeability. The nanoporous membranes may find application in a variety of filtering applications. ${ }^{83} \mathrm{Fu}$ et al. demonstrate the use of graphene oxide liquid crystals can be applied as composite inks for the formation of electrodes in 3D printing applications ${ }^{84}$ due to the intrinsic self-assembly that means they retain ordering of the GO platelets on drying of the solvent.

The development of fibers formed from graphene, $\mathrm{GO}$ or reduced GO is a widely reviewed maturing area for investigation, ${ }^{85-88}$ with many proposed applications such as in conducting wires,
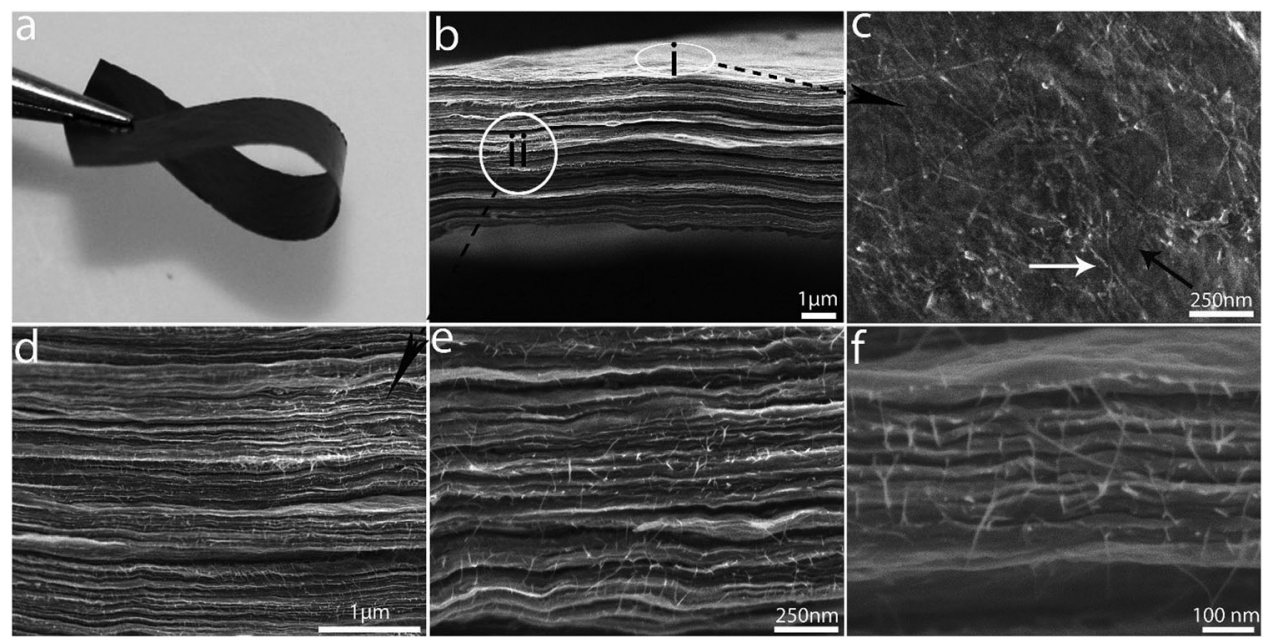

Fig. 6 (a) Photograph of a flexible free-standing paper of LC GO made by a cast drying method. (b) SEM image of the cross section of as-cast dried LC GO paper. (c) SEM image of the surface of the layer-by-layer composite, marked as region (i) in (b). (d-f) Cross section of composite paper at different. Reproduced with permission from Jalili et al., ACS Nano, $2013^{78}$ (C)2013 American Chemical Society). 

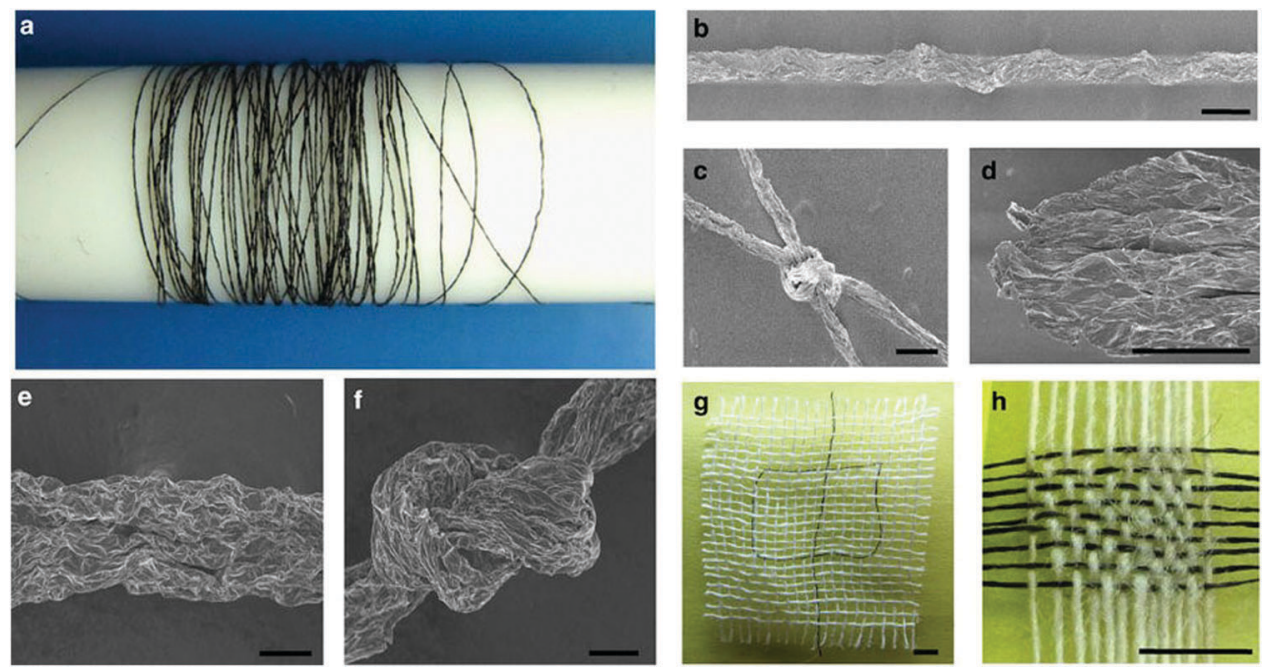

Fig. 7 (a) Four-metre-long wound GO fibre. SEM images of the fibre (b), and a typical tighten knot (c). (d) The morphology of the GO fibre after tensile tests. All scale bars $50 \mu \mathrm{m}$. Reproduced with permission from Xu and Gao, Nat. Commun., $2011^{4}$ (C)2011 Nature Publishing Group).

energy storage and conversion devices, actuators, field emitters, catalysis and optoelectronic and photonic devices. One of the most promising developments in this field, and of particular interest here, has been the use of the liquid crystal phase to improve the homogeneity and ordering of the fibers produced; numerous examples exist where fibers comprised of $2 \mathrm{D}$ materials have also been produced by the wet-spinning of liquid crystalline solutions. ${ }^{4,47,49,50,89} \mathrm{Xu}$ and $\mathrm{Gao}^{4}$ developed a method by which aqueous graphene oxide liquid crystals were continuously spun into metres of macroscopic graphene oxide fibres; subsequent chemical reduction gave the first macroscopic neat graphene fibres with high conductivity and good mechanical performance (Fig. 7). Jalili et al. demonstrate a method for one-step continuous spinning of graphene fibers where the need for post-treatment processes is eliminated by the use of basic coagulation baths for reduction of GO during the spinning process, ${ }^{49}$ as well as the applicability of wet-spinning to the formation of fibers of other $2 \mathrm{D}$ materials. ${ }^{50}$

\section{Optoelectronics}

Liquid crystalline nanocomposites incorporating 2D material particles show great promise for optoelectronic applications due to their field induced tunability and enhanced functionality stemming from the plethora of properties displayed by the range of exfoliatable materials. For example, dispersions of liquid crystalline graphene oxide have been shown to undergo electro-optical switching with low threshold voltage requirements. ${ }^{90}$ Kim et al. show that GO LCs possess an extremely large Kerr coefficient, making them attractive for low power consumption optoelectronic devices. By stabilising a suspension of reduced GO using surfactants, they demonstrated increased time stability and drastically improved electro-optic properties with an induced birefringence twice as large at the same field strength as that with an unreduced GO suspension.

Zhu et al. ${ }^{91}$ have shown that the preparation of $\operatorname{poly}(\mathrm{N}$ isopropylacrylamide)/GO nanocomposite hydrogels with macroscopically oriented LC structures, after polymerisation, can be readily achieved under assistance from a flow-field - induced by vacuum degassing. Nanocomposites prepared with a GO concentration of $5.0 \mathrm{mg} \mathrm{mL}{ }^{-1}$ exhibit macroscopically aligned LC structures, which endow the gels with anisotropic optical properties. Furthermore, they show that the oriented LC structures are not damaged during switching of the hydrogels, and hence their behaviour undergoes reversible changes. Additionally, they show that the oriented LC structures in the hydrogels can be permanently maintained after drying the nanocomposite samples. The liquid crystalline properties of such nanocomposites facilitate their applicability to switching in optoelectronic devices.

Kim et al. ${ }^{92}$ have demonstrated significant improvement of the electro-optic performance of a polymer-stabilized liquid crystalline blue phase using a reduced graphene oxide (RGO) enriched polymer network. The conductivity of the nanocomposite system is increased by the inclusion of the RGO. Furthermore, reductions in the operational voltage $(\sim 32 \%)$, response time $(\sim 51 \%)$ and hysteresis $(\sim 53 \%)$ compared to that of a conventional polymer-stabilized BPLC signify great potential for the use of $2 \mathrm{D}$ materials in enhancing novel electrooptic device applications of conventional LC systems.

Recently, Hogan et al. proposed that by tuning the liquid crystal director by means of an applied field, one could induce the formation of metastructures formed of the dispersed $2 \mathrm{D}$ material particles as they are repositioned. In particular, they show that nanocomposites of nematic phase liquid crystals with dispersed graphene oxide particles can be integrated with CMOS photonics devices as a back-end process as part of microfluidic systems and that the integrated nanocomposites can be readily controlled by use of either an electric field or laser light to reposition and rearrange the dispersed particles ${ }^{3}$ (Fig. 8). They present a novel characterisation method based on Raman spectroscopy to allow determination of the spatial positioning of the integrated 2D material particles, allowing precise monitoring of metastructure formation. 


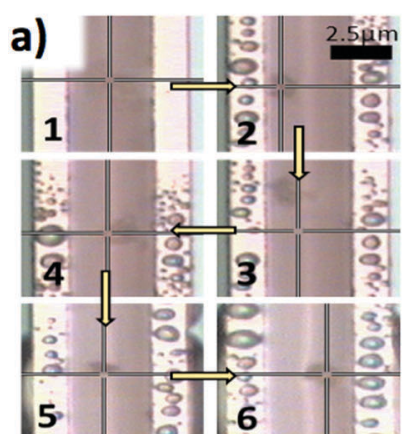

\section{b)}
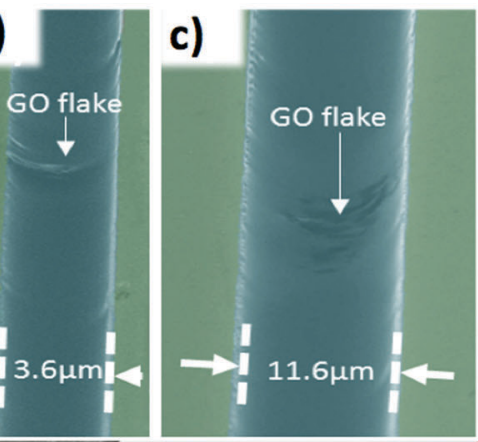

d)

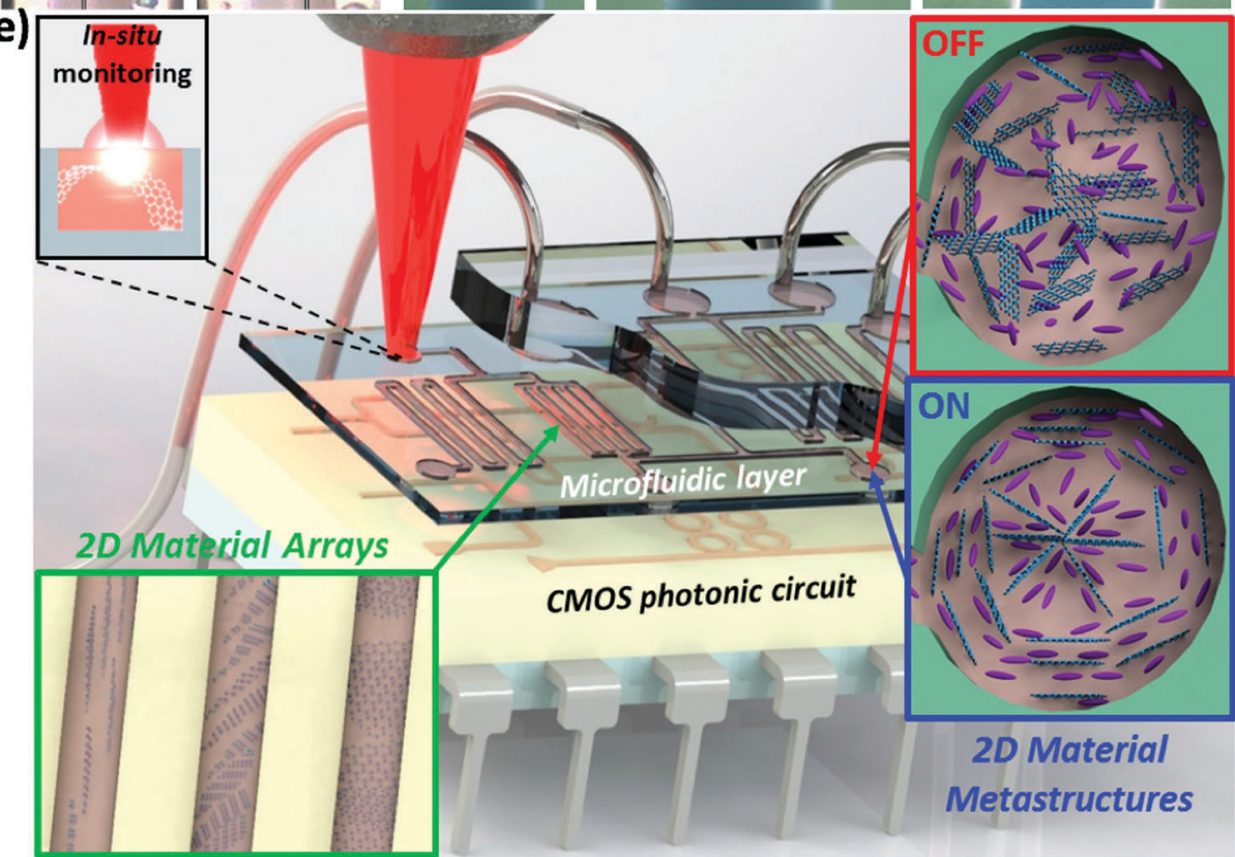

Fig. 8 (a) Control of liquid crystal dispersed 2D material particle using laser light. (b and c) SEM images of GO flakes integrated in microfluidic channels with a nematic liquid crystal host. (d) SEM image of GO flakes integrated into a microfluidic waveguide after removal of host fluid. (e) A CMOS photonic circuit coupled to a microfluidic layer integrating dynamically reconfigurable 2D material metastructures by exploiting liquid crystal technology. Adapted with permission from Hogan et al., Sci. Rep., $2017^{3}$ (C)2017 Nature Publishing Group).

\section{Displays}

2D material liquid crystals can be used in back-illuminated liquid crystal display applications as they exhibit electro-optic switching. The large Kerr coefficient of graphene oxide liquid crystals observed by Shen et al.,$^{53}$ for example, facilitates this application. However, the slow switching times reported by Kim and $\operatorname{Kim}(>1 \mathrm{~s})^{93}$ must be considered, although Ahmad et al. ${ }^{94}$ report that this can be improved by approximately an order of magnitude by careful selection of the size of graphene oxide mesogens.

More promisingly, 2D material liquid crystals have also been proposed for application in liquid crystal displays - particularly in so-called 'e-ink' displays - without requiring the polarising optics typically necessary for these applications. ${ }^{52,76} \mathrm{He}$ et al. ${ }^{52}$ demonstrate a process by which graphene oxide liquid crystals can be used for reflective displays without the need for polarizing optics (Fig. 9). By using flow-induced mechanical alignment, they prepared graphene oxide in different orientational orders and demonstrated that the ordered graphene oxide liquid crystals can be used as a rewritable display medium. The surface of the graphene oxide liquid crystal can be switched from a bright, reflective state to a dark, transmissive state using, for example, a wire to manually draw patterns on the surface. They explain that the contrast between the two states arises due to the anisotropic response of the flakes due to the inherent high aspect ratio of the $2 \mathrm{D}$ material.

\section{Quality control}

Inducing the onset of a liquid crystal phase in a dispersion of graphene oxide has been used for size selection of the graphene oxide particles. ${ }^{95}$ Lee $e t$ al. introduce a method for facile size selection of large-size graphene oxide particles by exploiting liquid crystallinity. They show that in a biphasic graphene oxide dispersion where both isotropic and liquid crystalline phases are in equilibrium, large-size GO flakes $(>20 \mu \mathrm{m})$ are spontaneously concentrated within the liquid crystalline phase. Selectivity of large flake sizes without the need of filtering presents several advantages for photonics and optoelectronics applications; 

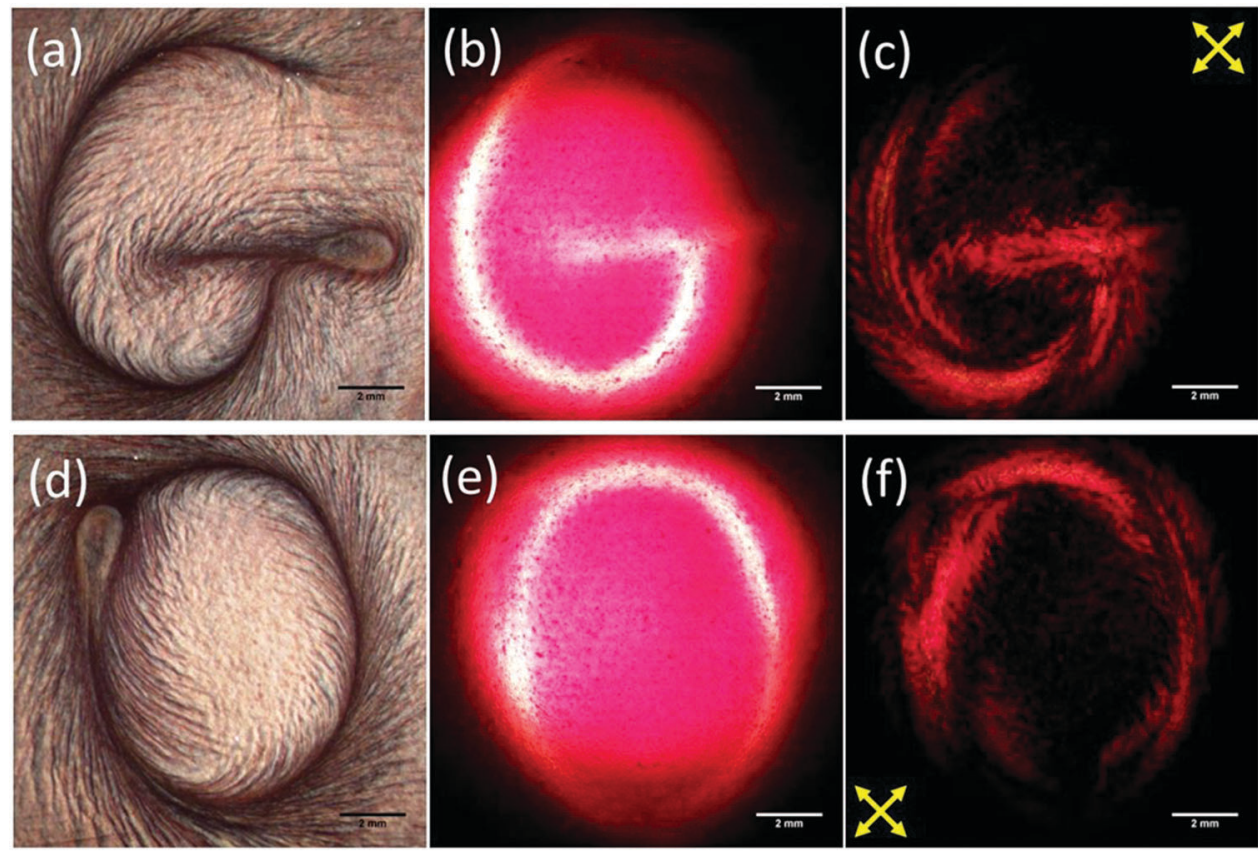

Fig. 9 Images of a defined structure in a liquid crystalline e-ink of graphene oxide dispersed in water in (a and d) reflection with unpolarised light, (b and e) transmission with unpolarised light and (c and f) transmission between crossed polarisers. Reproduced from He et al., Nanoscale, $2014^{52}$ with permission from The Royal Society of Chemistry (C2014.

primarily larger flakes allow for greater uniformity of device characteristics over wider areas and can help to increase the uniformity of depositions.

\section{Outlook}

2D materials encompass a fascinating range of diverse properties with a myriad of possible applications in optoelectronics and photonics. The development of liquid crystalline nanocomposite materials incorporating 2D materials represents a significant advance in the opportunities for integration and exploitation of 2D materials within these fields. However, there remain a large number of questions that demand further investigation before 2D material liquid crystals can find wider application. Primarily, there remain many candidate $2 \mathrm{D}$ materials for which a liquid crystal phase is theoretically possible but not yet shown; the discovery of further 2D material liquid crystals would broaden the range of utilisable properties available. Similarly to that observed for graphene oxide, observation of this liquid crystallinity should require a combination of careful solvent selection, tuning of the $2 \mathrm{D}$ material particle sizes and control of the concentration of the particles. Additionally, the use of surfactant molecules may be necessary to stabilise the liquid crystalline phase of the dispersions by maximising the aligning forces acting on the dispersed particles. However, this raises the additional question of the exploration - both theoretical and experimental - of the conditions required for the existence of the liquid crystal phase, an area in which little work has far been explored for the specific systems of interest here. A significant part of such work remains to be done in the comparison of the different synthetic routes towards the LC phase, and how the synthesis can affect the observed properties.
Additionally, dispersion of 2D materials in conventional liquid crystal host fluids presents superb new possibilities in optofluidic systems; from light generation to dynamic sensing applications. This is owing to the dramatic improvements that can be observed in the operational parameters of the nanocomposite systems in comparison to the conventional LC systems currently used in optoelectronics and photonics. Such nanocomposites, can not only improve properties such as switching times and threshold voltages, but can also add further functionality, for example by metastructuring of nanoparticle dispersions. For these nanocomposite systems, the most important advances to be made are in the fundamental understanding of the basis for improvements in their intrinsic properties; and in the exploration of predicting metastructuring as well as experimental observation.

Overall, the existence of liquid crystal phase 2D material dispersions presents fantastic opportunities in the exploration of novel optoelectronic and photonic systems, allowing new highly-scalable production processes for thin film integration and novel fiber systems amongst numerous other applications.

\section{Acknowledgements}

We acknowledge financial support from the Engineering and Physical Sciences Research Council (EPSRC) of the United Kingdom via the EPSRC Centre for Doctoral Training in Electromagnetic Metamaterials (Grant No. EP/L015331/1) and via Grant No. EP/N035569/1, EP/G036101/1 and EP/M002438/1. 


\section{References}

1 K. S. Novoselov, A. K. Geim, S. V. Morozov, D. Jiang, Y. Zhang, S. V. Dubonos, I. V. Grigorieva and A. A. Firsov, Science, 2004, 306, 666-669.

2 S. Li, M. Fu, H. Sun, Y. Zhao, Y. Liu, D. He and Y. Wang, J. Phys. Chem. C, 2014, 118, 18015-18020.

3 B. T. Hogan, S. A. Dyakov, L. J. Brennan, S. Younesy, T. S. Perova, Y. K. Gun'ko, M. F. Craciun and A. Baldycheva, Sci. Rep., 2017, 7, 42120.

4 Z. Xu and C. Gao, Nat. Commun., 2011, 2, 571.

5 C. Zakri, C. Blanc, E. Grelet, C. Zamora-Ledezma, N. Puech, E. Anglaret and P. Poulin, Philos. Trans. R. Soc., A, 2013, 371, 20120499.

6 S. Chandrasekhar, Liquid Crystals, Cambridge University Press, Cambridge, 1992.

7 P. G. de Gennes and J. Prost, The Physics of Liquid Crystals, Oxford University Press, New York, 2nd edn, 1995.

8 F. Reinitzer, Liq. Cryst., 1989, 5, 7-18.

9 F. Reinitzer, Monatsh. Chem., 1888, 9, 421-441.

10 J. Planer, Ann. Chem. Pharm., 1861, 118, 25-27.

11 A. Trokhymchuk, Condens. Matter Phys., 2010, 13, 1-4.

12 T. J. Sluckin, D. A. Dunmur and H. Stegemeyer, Crystals that flow: classic papers from the history of liquid crystals, Taylor \& Francis, 2004.

13 J. A. Castellano, Liquid Gold, World Scientific, 2005.

14 R. H. Chen, Liquid crystal displays: fundamental physics and technology, Wiley, 2011.

15 H.-S. Kwok, S. Naemura and H. L. Ong, Progress in Liquid Crystal Science and Technology, World Scientific, 2013.

16 S. Y. Vetrov, M. V. Pyatnov and I. V. Timofeev, Phys. Rev. E: Stat., Nonlinear, Soft Matter Phys., 2014, 90, 1-10.

17 D. C. Zografopoulos, R. Asquini, E. E. Kriezis, A. d'Alessandro and R. Beccherelli, Lab Chip, 2012, 12, 3598.

18 P. Li, M. Wong, X. Zhang, H. Yao, R. Ishige, A. Takahara, M. Miyamoto, R. Nishimura and H.-J. Sue, ACS Photonics, 2014, 1, 79-86.

19 V. A. Tolmachev, S. A. Grudinkin, J. A. Zharova, V. A. Melnikov, E. V. Astrova and T. S. Perova, in Proceedings of SPIE, ed. G. C. Righini, S. K. Honkanen, L. Pavesi and L. Vivien, 2008, vol. 6996, p. $69961 \mathrm{Z}$.

20 Q. Li, Nanoscience with Liquid Crystals, Springer International Publishing, Cham, 2014.

21 D. Donisi, B. Bellini, R. Beccherelli, R. Asquini, G. Gilardi, M. Trotta and A. D'Alessandro, IEEE J. Quantum Electron., 2010, 46, 762-768.

22 A. D’Alessandro and R. Asquini, Mol. Cryst. Liq. Cryst., 2003, 398, 207-221.

23 X. Li, C. W. Magnuson, A. Venugopal, J. An, J. W. Suk, B. Han, M. Borysiak, W. Cai, A. Velamakanni, Y. Zhu, L. Fu, E. M. Vogel, E. Voelkl, L. Colombo and R. S. Ruoff, Nano Lett., 2010, 10, 4328-4334.

24 A. Reina, X. Jia, J. Ho, D. Nezich, H. Son, V. Bulovic, M. S. Dresselhaus and J. Kong, Nano Lett., 2009, 9, 30-35.

25 Z. Sun, Z. Yan, J. Yao, E. Beitler, Y. Zhu and J. M. Tour, Nature, 2010, 468, 549-552.
26 D. Wei, Y. Liu, Y. Wang, H. Zhang, L. Huang and G. Yu, Nano Lett., 2009, 9, 1752-1758.

27 N. Behabtu, J. R. Lomeda, M. J. Green, A. L. Higginbotham, A. Sinitskii, D. V. Kosynkin, D. Tsentalovich, A. N. G. Parra-Vasquez, J. Schmidt, E. Kesselman, Y. Cohen, Y. Talmon, J. M. Tour and M. Pasquali, Nat. Nanotechnol., 2010, 5, 406-411.

28 Y. Hernandez, V. Nicolosi, M. Lotya, F. M. Blighe, Z. Sun, S. De, I. T. McGovern, B. Holland, M. Byrne, Y. K. Gun'Ko, J. J. Boland, P. Niraj, G. Duesberg, S. Krishnamurthy, R. Goodhue, J. Hutchison, V. Scardaci, A. C. Ferrari and J. N. Coleman, Nat. Nanotechnol., 2008, 3, 563-568.

29 M. Lotya, P. J. King, U. Khan, S. De and J. N. Coleman, ACS Nano, 2010, 4, 3155-3162.

30 A. O'Neill, U. Khan, P. N. Nirmalraj, J. Boland and J. N. Coleman, J. Phys. Chem. C, 2011, 115, 5422-5428.

31 F. Bonaccorso and Z. Sun, Opt. Mater. Express, 2014, 4, 63. 32 I. Ogino, Y. Yokoyama, S. Iwamura and S. R. Mukai, Chem. Mater., 2014, 26, 3334-3339.

33 J. I. Paredes, S. Villar-Rodil, A. Martínez-Alonso and J. M. D. Tascón, Langmuir, 2008, 24, 10560-10564.

34 X. Qi, T. Zhou, S. Deng, G. Zong, X. Yao and Q. Fu, J. Mater. Sci., 2014, 49, 1785-1793.

35 L. Zhang, J. Liang, Y. Huang, Y. Ma, Y. Wang and Y. Chen, Carbon, 2009, 47, 3365-3368.

36 L. Peng, Z. Xu, Z. Liu, Y. Wei, H. Sun, Z. Li, X. Zhao and C. Gao, Nat. Commun., 2015, 6, 5716.

37 D. W. Kim, D. Kim, B. H. Min, H. Lee and H.-T. Jung, Carbon, 2015, 88, 126-132.

38 X. Sun, D. Luo, J. Liu and D. G. Evans, ACS Nano, 2010, 4, 3381-3389.

39 T.-Z. Shen, S.-H. Hong and J.-K. Song, Carbon, 2014, 80, 560-564.

40 W. Zhang, X. Zou, H. Li, J. Hou, J. Zhao, J. Lan, B. Feng and S. Liu, RSC Adv., 2015, 5, 146-152.

41 X. Wang, H. Bai and G. Shi, J. Am. Chem. Soc., 2011, 133, 6338-6342.

42 T. Brettschneider, C. Dorrer, M. Bründel, R. Zengerle and M. Daub, J. Micromech. Microeng., 2013, 23, 55005.

43 S.-H. Hong, T.-Z. Shen and J.-K. Song, J. Phys. Chem. C, 2014, 118, 26304-26312.

44 B. Dan, N. Behabtu, A. Martinez, J. S. Evans, D. V. Kosynkin, J. M. Tour, M. Pasquali and I. I. Smalyukh, Soft Matter, 2011, 7, 11154.

45 S. H. Aboutalebi, M. M. Gudarzi, Q. Bin Zheng and J.-K. Kim, Adv. Funct. Mater., 2011, 21, 2978-2988.

46 M. M. Gudarzi, M. H. M. Moghadam and F. Sharif, Carbon, 2013, 64, 403-415.

47 Z. Xu and C. Gao, ACS Nano, 2011, 5, 2908-2915.

48 J. E. Kim, T. H. Han, S. H. Lee, J. Y. Kim, C. W. Ahn, J. M. Yun and S. O. Kim, Angew. Chem., Int. Ed., 2011, 50, 3043-3047.

49 R. Jalili, S. H. Aboutalebi, D. Esrafilzadeh, R. L. Shepherd, J. Chen, S. Aminorroaya-Yamini, K. Konstantinov, A. I. Minett, J. M. Razal and G. G. Wallace, Adv. Funct. Mater., 2013, 23, 5345-5354. 
50 R. Jalili, S. Aminorroaya-Yamini, T. M. Benedetti, S. H. Aboutalebi, Y. Chao, G. G. Wallace and D. L. Officer, Nanoscale, 2016, 8, 16862-16867.

51 R. Jalili, S. H. Aboutalebi, D. Esrafilzadeh, K. Konstantinov, J. M. Razal, S. E. Moulton and G. G. Wallace, Mater. Horiz., 2014, 1, 87-91.

52 L. He, J. Ye, M. Shuai, Z. Zhu, X. Zhou, Y. Wang, Y. Li, Z. Su, H. Zhang, Y. Chen, Z. Liu, Z. Cheng and J. Bao, Nanoscale, 2015, 7, 1616-1622.

53 T.-Z. Shen, S.-H. Hong and J.-K. Song, Nat. Mater., 2014, 13, 394-399.

54 Y. Zhu, S. Murali, W. Cai, X. Li, J. W. Suk, J. R. Potts and R. S. Ruoff, Adv. Mater., 2010, 22, 3906-3924.

55 D. C. Marcano, D. V. Kosynkin, J. M. Berlin, A. Sinitskii, Z. Sun, A. Slesarev, L. B. Alemany, W. Lu and J. M. Tour, ACS Nano, 2010, 4, 4806-4814.

56 D. R. Dreyer, S. Park, C. W. Bielawski and R. S. Ruoff, Chem. Soc. Rev., 2010, 39, 228-240.

57 Z. Liu, Y. Wang, X. Zhang, Y. Xu, Y. Chen and J. Tian, Appl. Phys. Lett., 2009, 94, 23-26.

58 X. Zheng, B. Jia, X. Chen and M. Gu, Adv. Mater., 2014, 26, 2699-2703.

59 K. P. Loh, Q. Bao, G. Eda and M. Chhowalla, Nat. Chem., 2010, 2, 1015-1024.

60 R. Ganatra and Q. Zhang, ACS Nano, 2014, 8, 4074-4099.

61 M. Xu, T. Liang, M. Shi and H. Chen, Chem. Rev., 2013, 113, 3766-3798.

62 K. Kobayashi and J. Yamauchi, Phys. Rev. B: Condens. Matter Mater. Phys., 1995, 51, 17085-17095.

63 H. Li, J. Wu, Z. Yin and H. Zhang, Acc. Chem. Res., 2014, 47, 1067-1075.

64 S.-W. Kang, S. Sprunt and L.-C. Chien, Adv. Mater., 2001, 13, 1179-1182.

65 R. Bitar, G. Agez and M. Mitov, Soft Matter, 2011, 7, 8198.

66 C. Blanc, D. Coursault and E. Lacaze, Liq. Cryst. Rev., 2013, 1, 83-109.

67 R. Jose, G. Skačej, V. S. S. Sastry and S. Žumer, Phys. Rev. E: Stat., Nonlinear, Soft Matter Phys., 2014, 90, 32503.

68 X. Wang, D. S. Miller, E. Bukusoglu, J. J. de Pablo and N. L. Abbott, Nat. Mater., 2015, 15, 106-112.

69 K. Higashiguchi, K. Yasui, M. Ozawa, K. Odoi and H. Kikuchi, Polym. J., 2012, 44, 632-638.

70 D. Pires, J.-B. Fleury and Y. Galerne, Phys. Rev. Lett., 2007, 98, 247801.

71 D. Coursault, J. Grand, B. Zappone, H. Ayeb, G. Lévi, N. Félidj and E. Lacaze, Adv. Mater., 2012, 24, 1461-1465.

72 Y. Li, E. Prince, S. Cho, A. Salari, Y. Mosaddeghian Golestani, O. D. Lavrentovich and E. Kumacheva, Proc. Natl. Acad. Sci. U. S. A., 2017, 114, 2137-2142.

73 L. Yu, Z. Cheng, Z. Dong, Y. Zhang and H. Yu, J. Mater. Chem. C, 2014, 2, 8501-8506.
74 M. A. Bates and D. Frenkel, J. Chem. Phys., 1999, 110, 6553.

75 B. Konkena and S. Vasudevan, J. Phys. Chem. C, 2014, 118, 21706-21713.

76 F. Lin, X. Tong, Y. Wang, J. Bao and Z. M. Wang, Nanoscale Res. Lett., 2015, 10, 435.

77 B. Senyuk, N. Behabtu, B. G. Pacheco, T. Lee, G. Ceriotti, J. M. Tour, M. Pasquali and I. I. Smalyukh, ACS Nano, 2012, 6, 8060-8066.

78 R. Jalili, S. H. Aboutalebi, D. Esrafilzadeh, K. Konstantinov, S. E. Moulton, J. M. Razal and G. G. Wallace, ACS Nano, 2013, 7, 3981-3990.

79 C. Bao, H. Zhang, C. A. Wilkie, S. Bi, X.-Z. Tang, J. Wu and J. Yang, Carbon, 2016, 107, 774-782.

80 S. Stankovich, R. D. Piner, X. Chen, N. Wu, S. T. Nguyen and R. S. Ruoff, J. Mater. Chem., 2006, 16, 155-158.

81 M. Supur, K. Ohkubo and S. Fukuzumi, Chem. Commun., 2014, 50, 13359-13361.

82 M. G. Nasab and M. Kalaee, $R S C A d v .$, 2016, 6, 45357-45368.

83 A. Akbari, P. Sheath, S. T. Martin, D. B. Shinde, M. Shaibani, P. C. Banerjee, R. Tkacz, D. Bhattacharyya and M. Majumder, Nat. Commun., 2016, 7, 10891.

84 K. Fu, Y. Wang, C. Yan, Y. Yao, Y. Chen, J. Dai, S. Lacey, Y. Wang, J. Wan, T. Li, Z. Wang, Y. Xu and L. Hu, Adv. Mater., 2016, 28, 2587-2594.

85 Y. Liu, Z. Xu, W. Gao, Z. Cheng and C. Gao, Adv. Mater., 2017, 29, 1606794.

86 R. Narayan, J. E. Kim, J. Y. Kim, K. E. Lee and S. O. Kim, Adv. Mater., 2016, 28, 3045-3068.

87 F. Meng, W. Lu, Q. Li, J.-H. Byun, Y. Oh and T.-W. Chou, Adv. Mater., 2015, 27, 5113-5131.

88 Z. Xu and C. Gao, Mater. Today, 2015, 18, 480-492.

89 S. Naficy, R. Jalili, S. H. Aboutalebi, R. A. Gorkin III, K. Konstantinov, P. C. Innis, G. M. Spinks, P. Poulin and G. G. Wallace, Mater. Horiz., 2014, 1, 326.

90 M. J. Kim, J. H. Park, J. Yamamoto, Y. S. Kim and G. Scalia, Phys. Status Solidi RRL, 2016, 10, 397-403.

91 Z. Zhu, Y. Li, H. Xu, X. Peng, Y.-N. Chen, C. Shang, Q. Zhang, J. Liu and H. Wang, ACS Appl. Mater. Interfaces, 2016, 8, 15637-15644.

92 M. Kim, R. K. Mishra, R. Manda, G. Murali, T.-H. Kim, M.-H. Lee, M. Yun, S. Kundu, B.-S. Kim, S. H. Lee, S.-T. Wu, A. Lien, J. Lu and Y. Su, RSC Adv., 2017, 7, 16650-16654.

93 J. Y. Kim and S. O. Kim, Nat. Mater., 2014, 13, 325-326.

94 R. T. M. Ahmad, S.-H. Hong, T.-Z. Shen and J.-K. Song, Opt. Express, 2015, 23, 4435.

95 K. E. Lee, J. E. Kim, U. N. Maiti, J. Lim, J. O. Hwang, J. Shim, J. J. Oh, T. Yun and S. O. Kim, ACS Nano, 2014, 8, 9073-9080.

96 M. Mitov, ChemPhysChem, 2014, 15, 1245-1250. 